\title{
CORRESPONDENCE
}

\section{Chronic respiratory care for neuromuscular diseases in adults}

\section{To the Editors:}

We read with interest the article describing noninvasive ventilation (NIV) alternatives to tracheostomy ventilation (TIV) [1]. We agree that NIV cannot be used indiscriminately, particularly for amyotrophic lateral sclerosis patients with severe bulbar involvement, and that nocturnal use can be beneficial [1]. However, up to continuous NIV can also be a viable alternative to TIV as has been demonstrated for over 500 patients with Duchenne muscular dystrophy, spinal muscular atrophy, polio, amyotrophic lateral sclerosis and other neuromuscular diseases, in many cases for 20 to $>50$ yrs of continuous ventilatory support $[2,3]$. In the section "NIV versus tracheostomy," AMBrosino et al. [1] stated that "tracheostomy ventilation may be preferred [...] when the patient is ventilator dependent for most of the day", but upon review of their references, we found no mention of the clear preference for NIV by continuously NIV dependent neuromuscular disease populations [4]. Of 168 patients who used NIV and TIV continuously, both for $\geqslant 1$ month, all who were decannulated to NIV preferred it for safety, speech, swallowing, cosmesis, sleep and, especially, overall, as did the majority of those who underwent tracheotomy after using NIV [4]. Since AMBrosino et al. [1] do not employ the most convenient, cosmetic and effective methods for daytime NIV, that is, ventilatory support via $15 \mathrm{~mm}$ angled mouthpieces (not widely available in Europe) kept near the mouth for the patient to grab three or four times a minute or exsufflation belts [2], rather than be hooked up to ventilators via nasal interfaces all day, their counselling is biased towards tracheotomy, as ours is to remain noninvasive. Thus, it is not surprising that their patients might consider tracheotomy preferable because they are told that it is inevitable. In yet another study, 157 intubated patients who failed extubations and spontaneous breathing attempts were told that survival was impossible without tracheotomy but refused it and remained intubated up to 76 days until they had the resources to be transferred to our unit for extubation without tracheotomy [5]. Their extraordinary determination to refuse tracheotomy when given a noninvasive alternative speaks for itself [5].

\section{A.J. Hon and J.R. Bach}

University of Medicine and Dentistry of New Jersey, New Jersey Medical School, Newark, NJ, USA.

Correspondence: A.J. Hon and J.R. Bach, University of Medicine and Dentistry of New Jersey, New Jersey Medical School, Newark, NJ, USA 07103. E-mail: honaj@umdnj.edu

Statement of Interest: None declared.

\section{REFERENCES}

1 Ambrosino N, Carpene N, Gherardi M. Chronic respiratory care for neuromuscular diseases in adults. Eur Respir J 2009; 34: 444-451.

2 Bach JR, Alba AS, Saporito LR. Intermittent positive pressure ventilation via the mouth as an alternative to tracheostomy for 257 ventilator users. Chest 1993; 103: 174-182.

3 Bach JR. The Management of Patients with Neuromuscular Disease. Philadelphia, Elsevier, 2004, pp. 289-296.

4 Bach JR. A comparison of long-term ventilatory support alternatives from the perspective of the patient and care giver. Chest 1993; 104: 1702-1706.

5 Bach JR, Goncalves MR, Hamdani I, et al. Extubation of patients with neuromuscular disease: a new management paradigm. Chest 2009; [Epub ahead of print DOI: 10.1378/chest.09-2144].

DOI: 10.1183/09031936.00200709

\section{From the authors:}

We would like to thank A.J. Hon and J.R. Bach for their interest in our article [1] and for their useful suggestions concerning management of these patients. We apologise that we neglected to cite the reference quoted in the paper [2].

They seem to suspect that we are fans of tracheostomy ventilation (TIV), always and in any way. We want to assure them that this is not the case. We made the best effort to review chronic respiratory care techniques on the basis of the present evidence. Nevertheless, the topic of noninvasive ventilation (NIV) versus TIV is actually a matter of experience rather than of evidence-based medicine. A.J. Hon and J.R. Bach support their conclusions about NIV versus TIV with studies performed exclusively by their own group [2-5]; no randomised controlled studies are cited. We appreciate and acknowledge their results in the acute setting but we wonder how these results apply to long-term (usually non-professionally managed) home therapy. We also wonder why, after almost two decades, no other group claims the superiority of one modality over another. Reproducibility of results is important for diffusion of techniques. Indeed, there is no agreement either on time of tracheostomy or on the possible impact of tracheostomy on survival: clinical protocols for tracheostomy are far from being standardised [6]. This is confirmed by an Italian survey on 719 patients from 32 Italian respiratory intermediate care units, which reports that a substantial proportion of patients maintained tracheostomy despite the fact that they did not require mechanical ventilation, with no agreement on indications and systems for closing tracheostomy [7]. This suggests the need to evaluate the choice of interface for patients on an individual basis.

The time for ideologies is over... 